Research Article

\title{
Kinetic Study of SN2 Reaction between Paranitrophenyl Benzoate and Hydrazine in the Presence of CTAB Reverse Micelles
}

\author{
K. Bhargavi ${ }^{1}$ P. Shyamala ${ }^{1 *}$, M. Padma ${ }^{1}$ K.V. Nagalakshmi ${ }^{2}$ \\ ${ }^{1}$ Department of PNCO, School of Chemistry, Andhra University, Visakhapatnam, 530017, Andhra \\ Pradesh, India. \\ ${ }^{2}$ Department of Chemistry, Gayatri Vidya Parishad College of Engineering (Autonomous), \\ Visakhapatnam, 530048, Andhra Pradesh, India.
}

Received: 20th May 2021; Revised: $3^{\text {rd }}$ August 2021; Accepted: $3^{\text {rd }}$ August 2021

Available online: 14th August 2021; Published regularly: December 2021

\section{Abstract}

Kinetic study of the reaction between p-Nitrophenyl benzoate (PNPB) by hydrazine (HYN) in the presence of Cetyltrimethylammonium bromide (CTAB)/Chloroform/Hexane reverse micellar medium shows that the reaction obeys first order kinetics with respect to each of the reactants. The rate of the reaction is much slower in reverse micellar medium compared to aqueous medium under identical conditions $\left(k_{A q}{ }_{A q}=2.84 \times 10^{-3} \mathrm{sec}^{-1}, k_{r m}=1.34 \times 10^{-4}\right.$ $\left.\mathrm{sec}^{-1}\right)$. The rate constants for the reaction in the reverse micellar medium have been determined at different values of $W\left\{W=\left[\mathrm{H}_{2} \mathrm{O}\right] /[\mathrm{CTAB}]\right\}$ and at different concentrations of CTAB. It was found that the observed rate constant decreases with $W$. This kinetic behaviour was interpreted by using modified Berezin pseudo phase model, taking into consideration the distribution of the reactants, PNPB and hydrazine between the three pseudo phases, i.e., water pool, interface an organic phase.

Copyright (C) 2021 by Authors, Published by BCREC Group. This is an open access article under the CC BY-SA License (https://creativecommons.org/licenses/by-sa/4.0).

Keywords: CTAB reverse micelles; Hydrazine; Kinetics; p-Nitrophenyl benzoate; Water pools

How to Cite: K. Bhargavi, P. Shyamala, M. Padma, K.V. Nagalakshmi (2021). Kinetic Study of SN2 Reaction between Paranitrophenyl Benzoate and Hydrazine in the Presence of CTAB Reverse Micelles. Bulletin of Chemical Reaction Engineering \& Catalysis, 16(4), 744-751 (doi:10.9767/bcrec.16.4.11036.744-751)

Permalink/DOI: https://doi.org/10.9767/bcrec.16.4.11036.744-751

\section{Introduction}

The surfactant aggregates formed spontaneously in organic solvents are called reverse micelles and water is readily solubilized in the polar core of reverse micelle forming a water pool, characterized by a parameter $W$ $\left\{W=\left[\mathrm{H}_{2} \mathrm{O}\right] /[\mathrm{CTAB}]\right\}[1-3]$. This solubilized water exhibits special properties like lower dielectric constant, higher ionic strength (in the case of reverse micelles made of ionic surfactants) and altered nucleophilicity compared to bulk water [48]. Since the water pool has different properties

* Corresponding Author.

Email: shyamalapulipaka@rediffmail.com (P. Shyamala) compared to ordinary water, the rates of reactions and mechanisms can be expected to be different.

Reverse micelles are known to solubilise both polar and non-polar solutes [9-12] and because of these unique properties they are widely used as reaction media to design variety of reactions and synthesize novel materials [13-15]. This media is also very useful for the synthesis of nanoparticles with desired shape and size [16-20]. Three reactions, kinetics of dissociation of $\left[\mathrm{Fe}(\mathrm{tptz})_{2}\right]^{2+}$, oxidation of $\left[\mathrm{Fe}(\text { phen })_{3}\right]^{2+}$, indigo carmine and toluidine blue by periodate in the presence of CTAB reverse micelles have been earlier reported from this laboratory [21-23]. In the kinetics of dissociation of $\left[\mathrm{Fe}(\mathrm{tptz})_{2}\right]^{2+}$, low 
dielectric constant of the water pool facilitates the formation of ion pair between complex and $\mathrm{Br}^{-}$ion of $\mathrm{CTAB}$ and leads to increase in rate of reaction. In the study of kinetics of oxidation of $\left[\mathrm{Fe}(\text { phen })_{3}\right]^{2+}$ in CTAB reverse micelles, kinetic results have been quantitatively explained based on ionic strength of the water pool using Guggenheim equation. In the study of kinetics of oxidation of Indigo Carmine by periodate in CTAB reverse micelles, it was found that water molecule exists in the rate determining step of the reaction mechanism and the observed kinetic results have been quantitatively explained using Berezin pseudo phase model. In order to further explore the applications of reverse micelles and to develop kinetic models for a variety of reactions, an organic reaction $\left(\mathrm{SN}^{2}\right.$ reaction) between Paranitrophenyl benzoate and hydrazine in CTAB reverse micellar medium, was chosen for study. The results of the kinetic study of this reaction along with the kinetic model are presented in this paper.

\section{Materials and Methods}

\subsection{Materials}

Stock solutions of PNPB (99\% pure, Merck, India) and hydrazine (99\% pure, Merck, India) were prepared in double distilled water. Chloroform and hexane were used after distillation. Stock solutions of CTAB (Cetyl tri methyl ammonium bromide, $98 \%$ pure, Sigma, India) were prepared in 3:2 (v/v) chloroform-hexane mixtures.

\subsection{Preparation of Reverse Micellar Medium and Initiation of the Reaction}

An amount of $0.04 \mathrm{~mL}$ of hydrazine solution of concentration $2.5 \mathrm{~mol} . \mathrm{dm}^{-3}$ was added into $10 \mathrm{~mL}$ of 0.1 mol.dm ${ }^{-3}$ CTAB solution using a

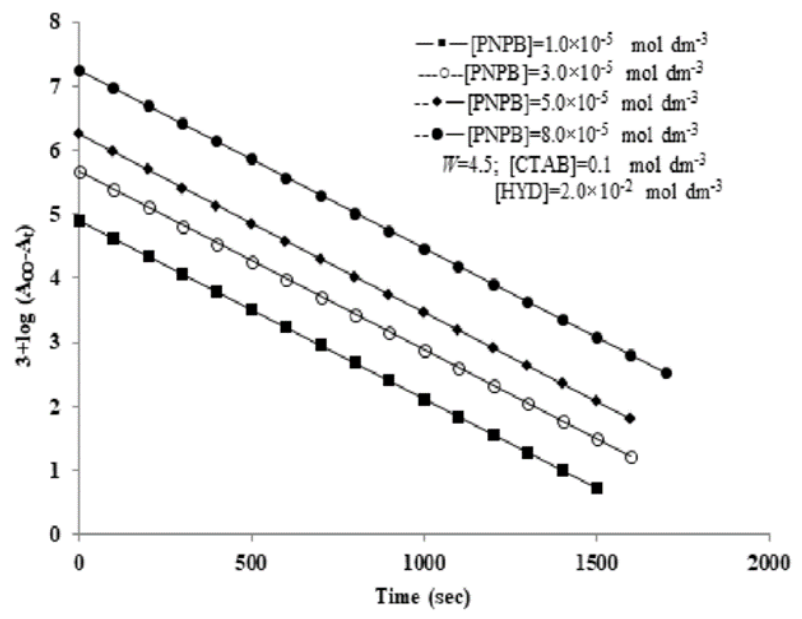

Figure 1. Plots of $\log \left(A_{{ }}-A_{t}\right)$ versus time. micro pipette. $0.02 \mathrm{~mL}$ of PNPB solution of concentration $0.02 \mathrm{~mol} . \mathrm{dm}^{-3}$ was then added to initiate the reaction. The reaction mixtures were shaken sufficiently to obtain a homogenous reverse micellar medium. The reaction was studied by changing the value of $W$ in the range 3.33 to 20.0 by varying the molar ratio of [Water] to [CTAB].

2.3 Experimental Method of Following the Reaction

The reaction was monitored by measuring the increase in absorbance of the product $p$ Nitro phenol where it has maximum absorbance at a wave length of $400 \mathrm{~nm}$, using a SHIMADZU UV-1800 double beam spectrophotometer. The reaction was carried out under pseudo first order conditions, [HYD] $>$ [PNPB] and the pseudo first order rate constants $k^{\prime}$ were obtained from plots of $\log \left(A_{\infty}-A_{t}\right)$ versus time. The second order rate constants, $k_{2}$, were obtained by dividing the pseudo first order rate constants, by the overall concentration of hydrazine. The kinetic data obtained are the averages from triplicate runs with reproducibility less than $\pm 4 \%$.

\section{Results and Discussion}

The kinetic investigations of the reaction have been carried out under the conditions, $\{[\mathrm{HYD}]>>[\mathrm{PNPB}]\}$, and plots of $\log \left(A_{\infty}-A_{t}\right)$ versus time were found to be good straight lines showing first order dependence with respect to PNPB (Figure 1). The pseudo first order rate constant $k^{\prime}$, was found to be directly proportional to concentration of hydrazine showing first order kinetics with respect to hydrazine (Figure 2). The reaction has also been carried out in aqueous medium under identical condi-

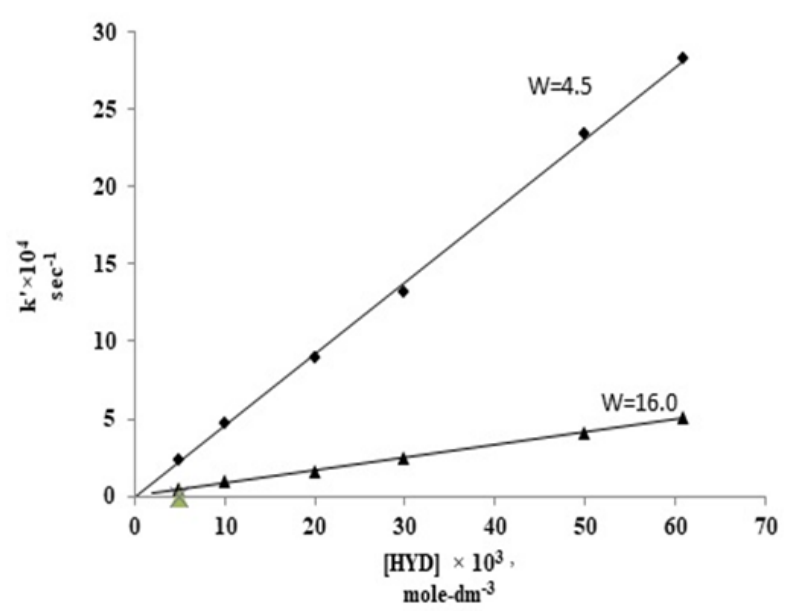

Figure 2. Plots of $k^{\prime}$ versus [HYD]。. 
tions and similar kinetic features were observed. The reaction is much faster in the presence of aqueous medium (approximately twenty five times) compared to CTAB reverse micellar medium (Table 1).

The slow rate of reaction in the presence of reverse micelles can be explained as follows: the reaction follows an $\mathrm{SN}^{2}$ mechanism and the transition state develops a partial negative charge compared to the reactants which are neutral (Scheme 1). Since high dielectric constant conditions favours such a transition state, the reaction is much faster in aqueous medium compared to CTAB reverse micelles.

\subsection{Effect of $W$ on Rate of Reaction}

Three different sets of experiments were conducted at three different concentrations of CTAB. At each fixed concentration of CTAB, a series of experiments were carried out at different values of $W$ (Table 2). It can be observed from the table that the pseudo first order rate constants $\mathrm{k}^{\prime}$, decrease with increase in $W$. Hydrazine is highly hydrophilic species and therefore can exist in the aqueous water pool and interface. Since PNPB is an organic compound, found to be distributed between the three pseu- do phases. $K_{H Y}$ and $K_{P N P B}$ represent distribution constants between the water pool and the interface and $Q_{P N P B}$ is the distribution constant between the water pool and the oil phase and are given by the equations;

$$
K_{H Y D}=\frac{[H Y D]_{s}}{[H Y D]_{w}} ; K_{P N P B}=\frac{[P N P B]_{s}}{[P N P B]_{w}} ; G_{P N P B}=\frac{[P N P B]_{o}}{[P N P B]_{w}}
$$

Accordingly, the mechanism shown in Scheme 2 is considered to explain the kinetic behaviour $[25,26]$.

In the reaction Scheme 2, the overall concentrations of hydrazine and PNPB are related to the corresponding local concentrations in

Table 1. Comparison of rate constants in the aqueous medium and in the presence of reverse micelles (0.1 mol. $\left.\mathrm{dm}^{-3} \mathrm{CTAB}\right)$ at same ionic strength $(\mu), \quad[\mathrm{HYD}]=2.0 \times 10^{-2} \mathrm{~mol} . \mathrm{dm}^{-3}$; $[\mathrm{PNPB}]=5.0 \times 10^{-5} \mathrm{~mol} . \mathrm{dm}^{-3} ; \mathrm{T}=304 \pm 0.1 \mathrm{~K}$.

\begin{tabular}{ccc}
\hline $\begin{array}{c}\mu \\
\left(\mathrm{mol} . \mathrm{dm}^{-3}\right)\end{array}$ & $\begin{array}{c}k_{\text {aq.med }}^{\prime} \times 10^{3} \\
\left(\mathrm{sec}^{-1}\right)\end{array}$ & $\begin{array}{c}k_{(\mathrm{rev} \mathrm{mic})}^{\prime} \times 10^{4} \\
\left(\mathrm{sec}^{-1}\right)\end{array}$ \\
\hline 3.46 & 2.84 & 1.34 \\
4.16 & 2.84 & 1.91 \\
\hline
\end{tabular}
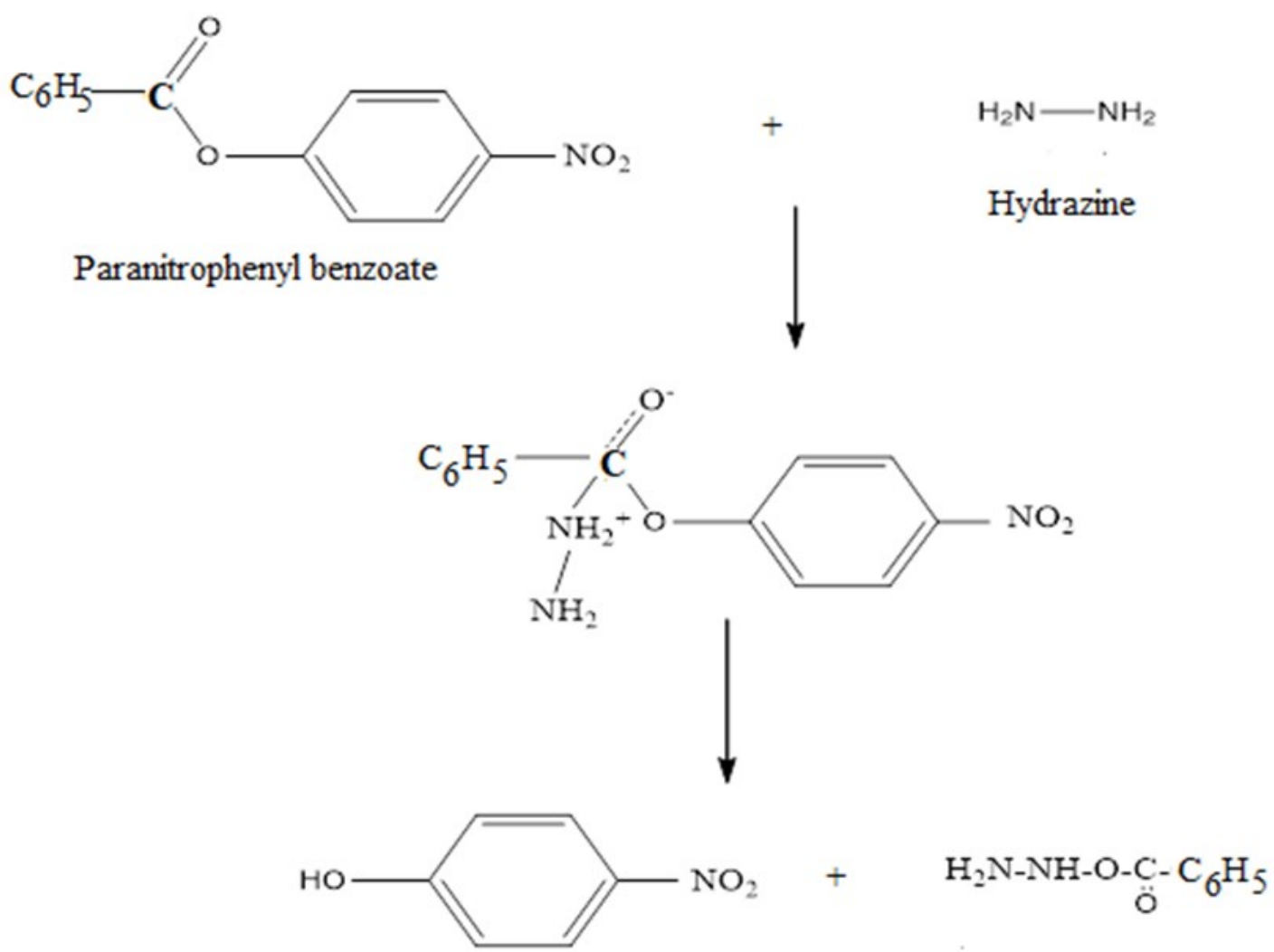

Benzoxyhydrazine

Scheme 1. Reaction mechanism 
each region by the mass balance Equations (2) and (3).

$$
\begin{aligned}
& {[H Y D]_{\text {OVR }}=[H Y D]_{w} \phi_{w}+[H Y D]_{s} \phi_{s}} \\
& {[P N P B]_{O V R}=[P N P B]_{w} \phi_{w}+[P N P B]_{s} \phi_{s}+[P N P B]_{o} \phi_{o}}
\end{aligned}
$$
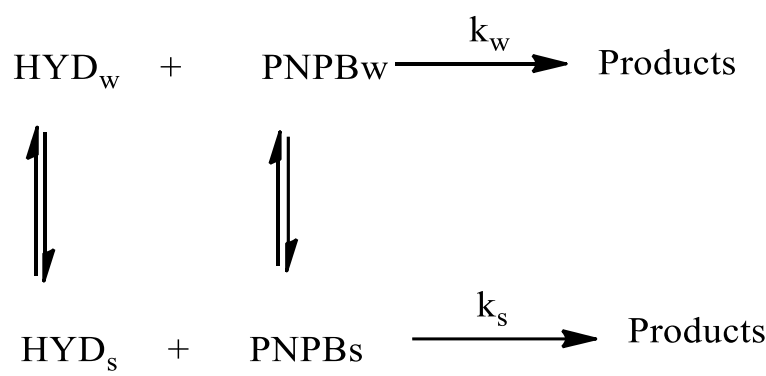<smiles>C=CC=C</smiles>

\section{$\mathrm{PNPB}_{\mathrm{o}}$}

Scheme 2. Distribution of reactants where,

$$
\begin{aligned}
\phi_{w} & =0.018[C T A B]_{o} W \\
\phi_{s} & =0.323[C T A B]_{o} \\
\phi_{0} & =1-\left(\phi_{w}+\phi_{s}\right)
\end{aligned}
$$

where, $\phi_{\mathrm{w}}, \phi_{\mathrm{s}}$ and $\phi_{\mathrm{o}}$ represent the volume fractions of the aqueous droplets, the interface and the oil phase, respectively. The overall second order rate constant, $k_{2}$ is given by Equation (7).

$$
\begin{aligned}
k_{2} & =\frac{k^{\prime}}{[H Y D]_{O V R}} \\
& =\frac{k_{w} \phi_{w}+k_{s} \phi_{s} K_{H Y D} K_{P N P B}}{\left(\phi_{w}+K_{H Y D} \phi_{s}\right)\left(k_{w} \phi_{w}+k_{s} \phi_{s} K_{H Y D} K_{P N P B}\right)}
\end{aligned}
$$

Equation (7) can be simplified by assuming that the hydrophobic PNPB reside preferentially in the oil phase compared to aqueous pools and that $\phi_{0}$ is larger than either $\phi_{\mathrm{w}}$ and $\phi_{\mathrm{s}}$. As a

\begin{tabular}{|c|c|c|c|c|}
\hline $\begin{array}{c}\text { CTAB } \\
\left(\mathrm{mol}^{\left.-d^{-3}\right)}\right.\end{array}$ & {$\left[\mathrm{Br}^{-}\right]_{\mathrm{e}}$} & $W$ & $\begin{array}{l}k^{\prime} \times 10^{4} \\
\left(\mathrm{sec}^{-1}\right)\end{array}$ & $\begin{array}{c}k_{2} \times 10^{2} \\
\left(=k^{\prime} /[\mathrm{HYD}]_{\mathrm{o}}\right)\end{array}$ \\
\hline \multirow[t]{8}{*}{0.1} & 22.2 & 2.5 & 20.0 & 10.0 \\
\hline & 15.6 & 3.5 & 13.6 & 6.80 \\
\hline & 12.1 & 4.5 & 8.92 & 4.46 \\
\hline & 8.47 & 6.5 & 5.81 & 2.90 \\
\hline & 6.53 & 8.5 & 4.10 & 2.05 \\
\hline & 4.16 & 13.0 & 1.91 & 0.95 \\
\hline & 3.46 & 16.0 & 1.34 & 0.67 \\
\hline & 2.77 & 20.0 & 0.92 & 0.46 \\
\hline \multirow[t]{8}{*}{0.2} & 22.2 & 2.5 & 19.2 & 9.6 \\
\hline & 15.6 & 3.5 & 13.4 & 6.7 \\
\hline & 12.1 & 4.5 & 8.80 & 4.4 \\
\hline & 8.47 & 6.5 & 5.43 & 2.71 \\
\hline & 6.53 & 8.5 & 3.80 & 1.9 \\
\hline & 4.16 & 13.0 & 1.78 & 0.89 \\
\hline & 3.46 & 16.0 & 1.28 & 0.64 \\
\hline & 2.77 & 20.0 & 0.86 & 0.43 \\
\hline \multirow[t]{8}{*}{0.3} & 22.2 & 2.5 & 20.0 & 10.0 \\
\hline & 15.6 & 3.5 & 13.2 & 6.60 \\
\hline & 12.1 & 4.5 & 8.64 & 4.32 \\
\hline & 8.47 & 6.5 & 5.63 & 2.81 \\
\hline & 6.53 & 8.5 & 3.0 & 1.5 \\
\hline & 4.16 & 13.0 & 1.82 & 0.91 \\
\hline & 3.46 & 16.0 & 1.32 & 0.66 \\
\hline & 2.77 & 20.0 & 1.04 & 0.52 \\
\hline
\end{tabular}
consequence, Equation (7) is reduced, after an appropriate rearrangement and by the use of Equation (4) to Equation (6). In this equation, the denominator involved the parameter $W$

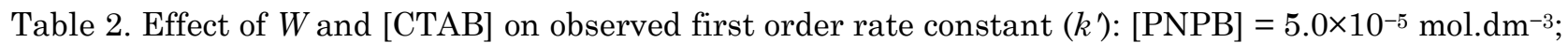
$[\mathrm{HYD}]=2.0 \times 10^{-2} \mathrm{~mol} . \mathrm{dm}^{-3} ; T=302 \mathrm{~K}$. 
which is important in determining the observed decreasing trend of $k_{2}$ with increasing molar ratio $W$.

$$
k_{2} \phi_{o}=\frac{1.80 \times 10^{-3} k_{w} W+k_{s} \phi_{s} K_{H Y D} K_{P N P B}}{Q_{P N P B}\left(1.80 \times 10^{-3} W+K_{H Y D} \phi_{s}\right)}
$$

The partition coefficient of hydrazine can be assumed to be much less than 1 since there is no exchange between hydrazine with the counter ions Br- of CTAB. Since the values of $\phi_{\mathrm{s}}$ lie in the range $0.89-0.95$ the term $K_{\mathrm{HYD}} \phi_{\mathrm{s}}$ can be neglected and Equation (8) now becomes,

$$
\begin{aligned}
& k_{2} \phi_{o}= \frac{\left(\frac{1.80 \times 10^{-3} k_{w}}{Q_{P N P B}}\right) W+\frac{k_{s} \phi_{s} K_{H Y D} K_{P N P B}}{Q_{P N P B}}}{\left(1.80 \times 10^{-3} W\right)} \\
& k_{2} \phi_{o}=\frac{k_{w}}{Q_{P N P B}}+\frac{k_{s} \phi_{s} K_{H Y D} K_{P N P B} / Q_{P N P B}}{\left(1.80 \times 10^{-3} W\right)}
\end{aligned}
$$

According to Equation (10), a plot of $k_{2} \phi_{0}$ versus $1 / W$ should be linear. Such a plot was obtained and Figure 3 shows that the plots of $k_{2} \phi_{0}$ versus $1 / W$ are good straight lines for 0.1 mol dm ${ }^{-3}$ of CTAB and is true for all concentrations of CTAB. At low values of $W(3.33-4.44)$ small deviation from linearity was observed. This linear trend implies the absence of special properties of the water pool and their effect on the reaction rate. Since the properties exist at $W<4$, there is a deviation from linear trend at low $W$ values.

\section{Conclusions}

The $\mathrm{SN}^{2}$ reaction of the PNPB by hydrazine obeys first order kinetics with respect to each of the reactants in aqueous as well as CTAB/Hexane/Chloroform reverse micellar me-

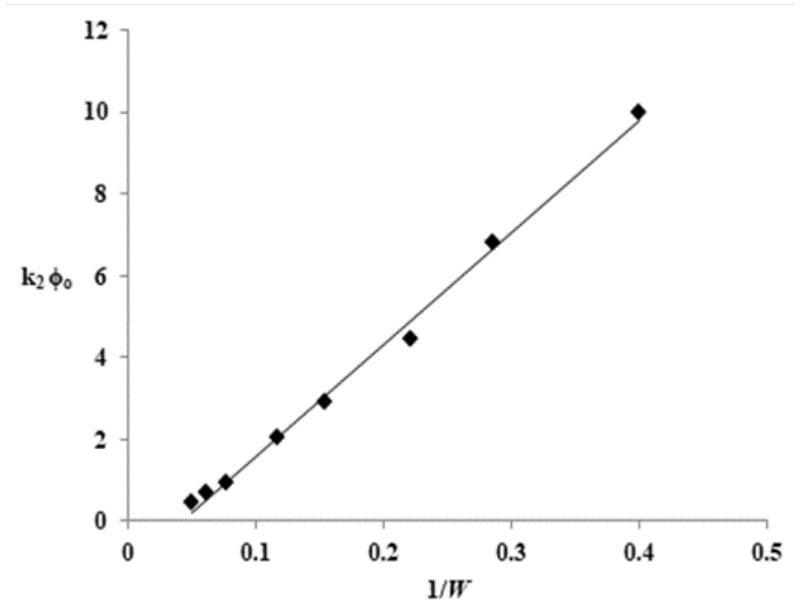

Figure 3. Plots of $k_{2} \phi_{\mathrm{o}}$ vs $1 / W$. dium. The reaction has been found to be inhibited around twenty times in the presence of CTAB reverse micelles compared to aqueous medium which can be attributed to low dielectric constant of water pool. The second order rate constant decreases with $W$ and is independent of CTAB concentration. The kinetic results are interpreted by applying Berezin pseudo phase model and accordingly the plots of $k_{2} \phi_{0}$ versus $1 / W$ are good straight lines for all concentrations of CTAB.

\section{Acknowledgements}

The authors (K. Bhargavi and P. Shyamala) are thankful to Ministry of Earth Sciences (MOES), National Center for Coastal Research (NCCR), India for financial support under major project No.MOES/ICMAM-PD/Supply Order/81/2017. K.V. Nagalakshmi is thankful to the management of Gayatri Vidya Parishad College of Engineering, Visakhapatnam for their support and encouragement.

\section{References}

[1] Michael, R., Branka, M.L, Nancy, E.L., Kenneth, W.H. (2004). Water motion in reverse micelles studied by quasielastic neutron scattering and molecular dynamics simulations. J. Chem. Phys., 121(16), 7855-7868. DOI: 10.1063/1.1792592.3.

[2] Amanda, J.M., John, W., Melanie, M.B. (2014). NMR and molecular dynamics study of the size, shape, and composition of reverse micelles in a cetyltrimethylammonium bromide (CTAB)/n-hexane/pentanol/water microemulsion. J. Phys. Chem. B, 118(36), 10767 10775. DOI: $10.1021 /$ jp504585k

[3] Lubica, K., Eva, M., Peter, S., Petr, S., Petr, K. (2015). Nature of CTAB/Water/Chloroform Reverse Micelles at Above- and Subzero Temperatures Studied by NMR and Molecular Dynamics Simulations. Langmuir, 31(30), $\begin{array}{lllllll}8 & 2 & 4 & - & 8 & 2 & 9\end{array}$ D O I : 10.1021/acs.langmuir.5b01776

[4] Mario, J.P., Chaimovich, H. (1986). Water activity in reversed sodium bis(2-ethylhexyl) sulfosuccinate micelles. J. Phys. Chem., 90(2), 282-287. DOI: 10.1021/j100274a016.

[5] Liu, Q. Gao, M., Zhang, J., Zhang, R., Li, J., Chen, S., Chen, G. (2020). Synthesis of interface activity of cetyl trimethylammonium benzoate, Russ. J. Phys. Chem. B, 14, 73-80. DOI: $10.1134 / \mathrm{S} 1990793120010066$

[6] Menger, F.M., Donohue J.A., Williams, R.F. (1973). Catalysis in water pools. J. Am. Chem. Soc., 95(1), 286-288. DOI: 10.1021/ja00782a075 
[7] Arindam, D., Animesh, P., Rajib Kumar, M. (2016). Modulation of anionic reverse micellar interface with non ionic surfactants can regulate enzyme activity within the micellar waterpool. Colloid and Polymer Science, 294, 715-726. DOI: 10.1007/s00396-016-38293

[8] Luisi, P.L., Straub, B.E. (1984). Reverse Micelles, Plenum Press, New York, P.73, DOI: 10.1002/pol.1985.130230312

[9] Pileni, M.P., (1993). Reverse micelles as micro reactors. J. Phys. Chem., 7, 6967-6973. DOI: 10.1021/j100129a008.

[10] Klyachko, N.L., Levashov, A.V., Kabanov, A.V. (1991). In: K Gratzel, K Kalyanasundaram (eds): Kinetics and Catalysis in Micro heterogeneous Systems. Marcel Dekker, New-York. 135-181

[11] Anjana, P., Ashutosh, P., (2008). Reverse micelles as suitable micro reactor for increased biohydrogen production, Int. J. Hydrogen Energy, $33(1), \quad 273-278$. D O : 10.1016/j.ijhydene.2007.07.013.

[12] Chopineau, J., Lagoutte, B., Thomas, D., Domurado, D. (2000) Reversed Micelles as Microreactors: N-terminal Acylation of RNase A and its Characterization. In: Gupta M.N. (eds) Methods in Non-Aqueous Enzymology. Methods and Tools in Biosciences and Medicine. Birkhäuser, Basel. DOI: 10.1007/978-30348-8472-3_10

[13] Irfan, H.L., Nagi, R.E.R., Jeenat, A., Arifa, A. (2019) Concept of Reverse Micelle Method For the Synthesis of Nano-Structured Materials. Current Nano Science, 15(2), 129-136. DOI: $10.2174 / 1573413714666180611075115$

[14] Hoorshad, F., James, P.K., Victor, R.V., Olivia, A.G. (2012). Ionic Concentration Effects on Reverse Micelle Size and Stability: Implications for the Synthesis of Nanoparticles. Langmuir, 25, $9267-9274$. DOI: $10.1021 / \mathrm{la} 300586 \mathrm{f}$

[15] Arash, H.K., Rashidi, A.M., Giti, K. (2017). Synthesis of tungsten nanoparticles by reverse micelle method. Journal of Molecular Liquids, 241, 897-903. DOI: 10.1016/j.molliq.2017.06.053.

[16] Garcia-Rio, L., Ramon Leis, J., Elena, P., Emilia, I. (1993). Transfer of the nitroso group in water/AOT/isooctane micro emulsions: intrinsic and apparent reactivity. J. Phys. Chem., 97(13), 3437-3442. DOI: 10.1021/j100115a057.
[17] Johnson, M.D., Lorenz, B.B., Wilkins, P.C., Lemons, B.G., Baruah, B., Lamborn, N., Stahla, M., Chatterjee, P.B., Richens, D.T., Crans, D.C. (2012). Switching Off Electron Transfer Reactions in Confined Media: Reduction of [Co(dipic)2]- and [Co(edta)]- by Hexacyanoferrate(II). Inorg. Chem., 51(5), 2757-2765. DOI: $10.1021 / \mathrm{ic} 201247 \mathrm{v}$.

[18] Chuanyi, Y., Shaokun, T., He, Z., Deng, X. (2005). Kinetics of lipase-catalyzed hydrolysis of olive oil in AOT/isooctane/reverse micelles. J. Mol. Catal. B Enzym., 35(4), 108-112. DOI: $10.1016 /$ j.molcatb.2005.06.005

[19] Miyake, Y., Owari, T., Ishiga, F., Teramoto, M. (1994). Enzymatic reaction in water-in-oil microemulsions - Rate of hydrolysis of a hydrophobic substrate, 2-naphthyl acetate. $J$. Chem. Soc., Faraday Trans., 90, 979-986. DOI: 10.1039/FT9949000979

[20] García-Río, L., Mejuto, J.C., Pérez-Lorenzo, M. (2005). Microheterogeneous Solvation for Aminolysis Reactions in AOT-Based Water-in-Oil Microemulsions. Chemistry A European Journal, 11(15), 4361-4373. DOI: 10.1002/chem.200401067.

[21] Nagalakshmi, K.V., Padma, M., Srikanth, V., Shyamala, P., Subba Rao, P.V. (2013). Catalytic effect of CTAB reverse micelles on the kinetics of dissociation of bis(2,4,6-tripyridyls-triazine) iron(II). Transition Met. Chem., 38, 523-527. DOI: 10.1007/s11243-013-9719-3.

[22] Nagalakshmi, K.V., Shyamala, P. (2019). Kinetics of oxidation of [Fe(phen)3]+2 by persulphate: catalysis in the water pools of CTAB reverse micelles. Bulg. Chem. Comm., 51(4), 494-498. DOI: 10.34049/bcc.51.4.4946.

[23] Nagalakshmi, K.V., Shyamala, P., Subba Rao, P.V. (2015). Catalytic effect of CTAB reverse micelles on the oxidation of indigo carmine by periodate, Ind. J. Chem., 54A, 351355.

[24] Nagalakshmi, K.V., Shyamala, P, Subba Rao, P.V. (2018). Kinetics of oxidation of toluidine blue by periodate: Catalysis by water pools of CTAB. Curr. Chem. Lett., 7, 93-100.

[25] Fletcher, P.D.I., Robinson, B.H. (1984). Effect of organised surfactant systems on the kinetics of metal ligand complex formation and dissociation. J. Chem. Soc. Faraday Trans., 80(1), 2417-2437.

[26] Schomacker, R., Stickdorn, K., Knoche, W. (1991). Chemical reactions in microemulsions: Kinetics of the alkylation of 2 alkylindan-1,3-diones in microemulsions and polar organic solvents. J. Chem. Soc., Faraday Trans., 87(6), 847-851. 


\section{APPENDICES}

A. Expression for Rate and Rate Constant

$$
\begin{aligned}
& \text { Rate }=k[H Y D]_{\text {OVR }}[P N P B]_{\text {OVR }} \\
& \text { Rate }=k^{\prime}[P N P B]_{\text {OVR }} \quad \text { where } k^{\prime}=k[H Y D]_{O V R} \quad(\text { PNPB is isolated }) \\
& k^{\prime}=\text { Rate } /[P N P B]_{\text {OVR }} \\
& \text { Rate }=k_{w} \phi_{w}[H Y D]_{w}[P N P B]_{w}+k_{s} \phi_{s}[P N P B]_{s}[H Y D]_{s} \\
& k^{\prime}=\frac{k_{w} \phi_{w}[H Y D]_{w}[P N P B]_{w}+k_{s} \phi_{s}[P N P B]_{s}[H Y D]_{s}}{[P N P B]_{O V R}} \\
& k^{\prime}=\frac{k_{w} \phi_{w}[H Y D]_{w}[P N P B]_{w}+k_{s} \phi_{s}[P N P B]_{s}[H Y D]_{s}}{[P N P B]_{w} \phi_{w}+[P N P B]_{s} \phi_{s}+[P N P B]_{o} \phi_{o}} \\
& k_{2}=\frac{k_{w}^{\prime} \phi_{w}[H Y D]_{w}[P N P B]_{w}+k_{s} \phi_{s}[P N P B]_{s}[H Y D]_{s}}{[H Y D]_{O V R}}=\frac{k_{w}}{\left\{[H Y D]_{w} \phi_{w}+[H Y D]_{s} \phi_{s}\right\}\left\{[P N P B]_{w} \phi_{w}+[P N P B]_{s} \phi_{s}+[P N P B]_{o} \phi_{o}\right\}}
\end{aligned}
$$

Dividing with $[H Y D]_{\mathrm{w}}[P N P B]_{\mathrm{w}}$ both numerator and denominator and replacing

$$
K_{H Y D}=\frac{[H Y D]_{s}}{[H Y D]_{w}} ; K_{P N P B}=\frac{[P N P B]_{s}}{[P N P B]_{w}} ; Q_{P N P B}=\frac{[P N P B]_{o}}{[P N P B]_{w}}
$$

We get

$$
k_{2}=\frac{k_{w} \phi_{w}+k_{s} \phi_{s} K_{H Y D} K_{P N P B}}{\left(\phi_{w}+K_{H Y D} \phi_{s}\right)\left(\phi_{w}+K_{P N P B} \phi_{s}+Q_{P N P B} \phi_{o}\right)}
$$




\section{B. Product of Reaction between Para Nitro Phenyl Benzoate and Hydrazine}

The product analysis was carried out by reacting $4.0 \times 10^{-5} \mathrm{~mol} . \mathrm{dm}^{-3}$ para nitrophenyl benzoate with equivalent amount of hydrazine in aqueous and CTAB reverse micellar medium. After completion of the reaction, the yellow coloured product obtained was subjected to column chromatography to separate the two products and then NMR and mass spectrum of the product was obtained (Figure S1 \& Figure S2). The spectra were found to be exactly identical with the spectra of para nitro phenol.

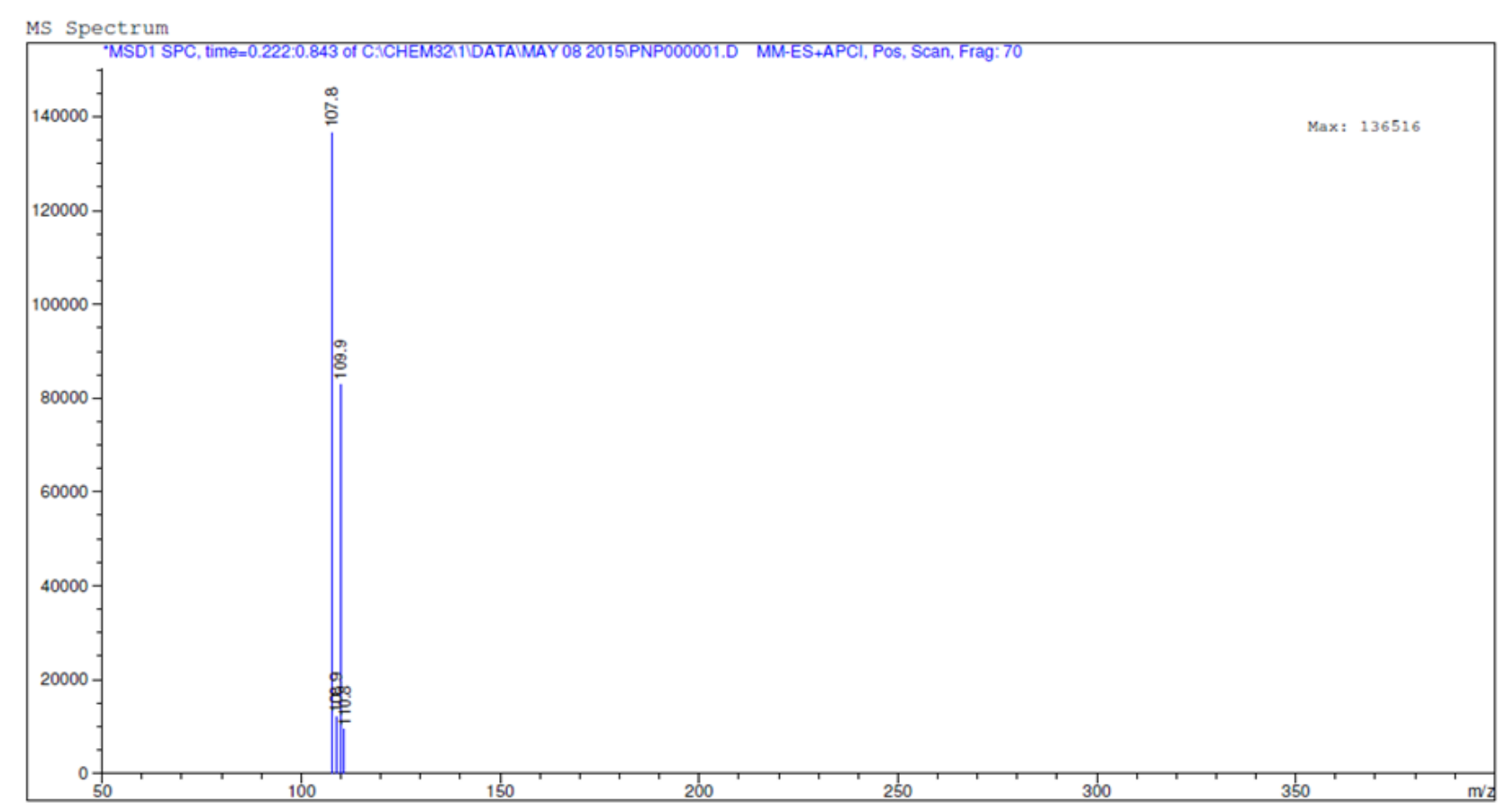

Figure S1. Product chromatogram

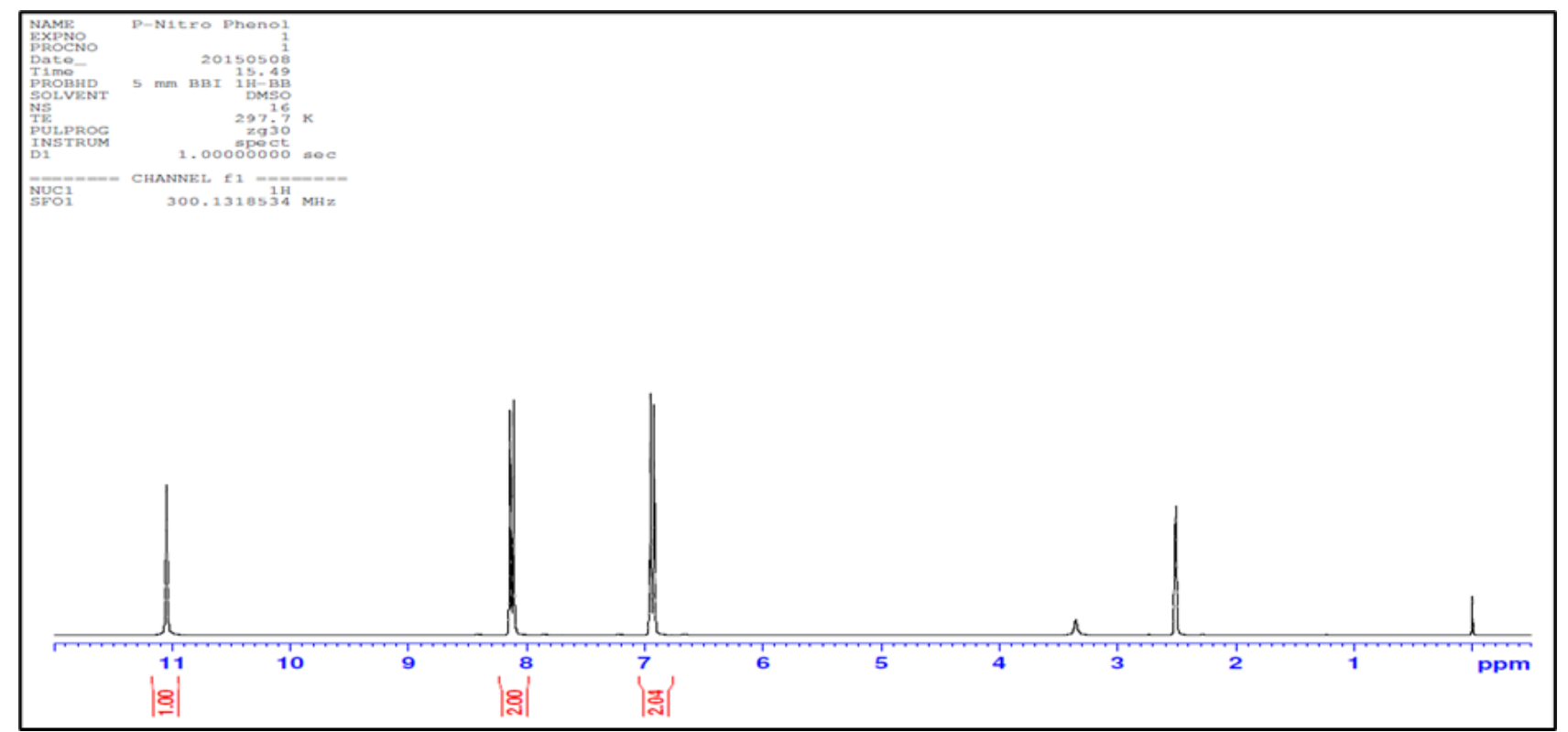

Figure S2. NMR mass spectrum of the product 